Research Article

\title{
Low-Vacuum Quadrupole Mass Filter Using a Drift Gas
}

\author{
Peihe Jiang $\mathbb{D}^{1,2}$ and Zhanfeng Zhao $\mathbb{D}^{3}$ \\ ${ }^{1}$ Shandong Fisherman Information Technology Co., Ltd., Weihai 264200, China \\ ${ }^{2}$ School of Opto-electronic Information Science and Technology, Yantai University, Yantai 264005, China \\ ${ }^{3}$ School of Information and Electrical Engineering, Harbin Institute of Technology, Harbin 150001, China \\ Correspondence should be addressed to Peihe Jiang; jiangpeihe@163.com
}

Received 26 August 2020; Accepted 12 December 2020; Published 29 December 2020

Academic Editor: Kevin Honeychurch

Copyright (c) 2020 Peihe Jiang and Zhanfeng Zhao. This is an open access article distributed under the Creative Commons Attribution License, which permits unrestricted use, distribution, and reproduction in any medium, provided the original work is properly cited.

\begin{abstract}
Performing mass spectrometry in a low-vacuum environment can markedly reduce the cost, size, and power consumption of instrumentation by reducing the workload of the pumping system. Under a low-vacuum environment, ions in a quadrupole mass filter do not have sufficient kinetic energy in the axial direction to reach the detector for mass analysis. To resolve this problem and develop a mass spectrometer suitable for a low-vacuum environment, a mass analysis method is proposed where a drift gas is used to supply energy to the ions. A simulation model was constructed in COMSOL Multiphysics, and a simple experimental device was built to validate the proposed method. The simulation results showed that this method effectively solves these problems, and the obtained spectral peak was superior to that without drift gas flow regarding spectral peak intensity and width. The experimental results showed that the proposed method separated ions with different mass-to-charge ratios at a pressure of $20 \mathrm{~Pa}$. This work provides a theoretical foundation for the development of low-vacuum mass spectrometry, which will promote portability, provide a lower threshold of use, and expand the fields of application for mass spectrometers.
\end{abstract}

\section{Introduction}

Performing mass spectrometry (MS) in a low-vacuum environment substantially reduces the cost, size, weight, and power of instrumentation and expands its applicability, making it more suitable for field applications $[1,2]$. This strategy is important for handheld mass spectrometers.

In recent years, with the development of mass analysers, ambient ion sources, atmospheric pressure interfaces, vacuum pumps, and electronic science, many remarkable achievements have been made with miniature mass spectrometers [3-6]. However, few studies have examined how to promote the portability of mass spectrometers from the perspective of reducing the level of vacuum required. Mass analysis in a low-vacuum environment can avoid the use of a bulky turbo pump and allow a handheld mass spectrometer to be developed. For a mass spectrometer, a high vacuum is typically necessary, and changes in pressure seriously affect performance [7]. For an ion trap, an increase in pressure cools the ions, which improves capture efficiency and resolution [8, 9]. However, as pressure increases, sample ions collide with the background gas molecules, which extends spectral peaks and lowers resolution and sensitivity, resulting in poor mass spectrometry results $[10,11]$. To our knowledge, no study has outlined the methods for mass analysis in a low-vacuum condition.

A quadrupole mass filter is one of the most mature and widely used mass analysers that consists of four parallel hyperboloid or cylindrical metal rods [12]. A quadrupole field is formed inside the mass filter using radio frequency (RF) voltages to the rods. After the sample is ionised using an ion source, the ions are accelerated via an electric field into the mass analyser with a specific initial velocity. Via filtration by the quadrupole field, ions with different mass-to-charge ratios are divided into two motion states: stable and unstable. Ions in stable motion can pass through the quadrupole analyser to reach the detector, and the ions in unstable motion hit the rods.

Based on the principle of the quadrupole mass filter, the motion of ions in the quadrupole can be decomposed into 
radial and axial components. Radial motion occurs in the direction of the cross section of rods, for which the quadrupole field supplies the energy to the ions. Axial motion is the motion parallel to the rods, for which the accelerating electric field supplies the energy to the ions. In general, when using a quadrupole mass filter, the pressure in the chamber must be maintained in the order of $10^{-5} \mathrm{~Pa}$. As background pressure increases, ions collide with the background gas molecules more frequently, and the energy of the ion's axial motion decreases gradually to zero. Thus, mass analysis will fail because the ions are unable to reach the detector located at the end of the analyser.

For a quadrupole mass filter, increased pressures can reduce kinetic energy along the ion's axial motion to levels that prevent analysis of the ions. To address this issue, a mass analysis method is proposed in this study where a drift gas flow is used to provide ejection energy to the ions. First, a simulation model based on this method was constructed in COMSOL Multiphysics. Then, a simple experimental platform was built to validate the proposed method.

\section{Method}

The core structure of the proposed low-vacuum mass analysis system consists of an ion source, a drift tube, a mass analyser, and an ion detector, as shown in Figure 1. The ion source is used to ionize the sample and is suitable for a highpressure environment. The drift tube is a place where drift air flows and is a semiclosed tubular structure with one end open and one closed end with a suction hole. The mass analyser is installed inside the drift tube, and a quadrupole mass filter with plate electrodes is used. A structurally optimised Faraday cup is used as the ion detector.

This core structure is placed in a vacuum chamber with a specified background gas pressure. The suction hole connects to a vacuum pump. When the vacuum pump is turned on, the drift gas flows in the same direction as the ions.

During mass analysis, the sample is ionised in the ion source. Then, the ions shoot into the mass analyser and travel under the combined action of the quadrupole field and the drift gas flow. The drift gas flow imparts kinetic energy to the ions for axial motion, and the quadrupole field selects ions depending on their mass-to-charge ratio in the cross-sectional direction. If the quadrupole electric field force is the dominant factor, the function of the mass filter can be described. Thus, although in a low-vacuum environment, ions in stable motion can pass through the mass analyser and hit the ion detector, which provides spectral information.

\section{Theoretical Modelling}

3.1. Quadrupole Mass Filter Based on Rectangular Electrodes. In the proposed mass analysis method, the axial movement of ions requires energy from the drift gas. Therefore, a closed airway with two ends open must be built for the drift gas to flow properly. A quadrupole filter based on rectangular electrodes [13] meets this requirement and is shown in Figure 2. Experimental results suggest that the quadrupole electric field remains dominated in this structure, allowing

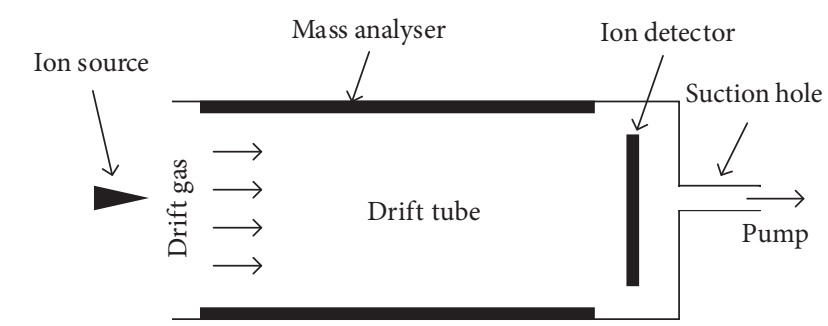

FIGURE 1: Schematic of the proposed low-vacuum mass analysis system.

mass analysis. In addition, the general mass filter with a hyperbolic or cylindrical electrode can also be used. However, a closed drift tube is required, and the mass analyser should be fixed inside it.

3.2. Ion Detector for the Homogeneous Drift Gas. A traditional Faraday ion detector is composed of a circular metal plate. When installed at the end of the drift tube, as is shown in Figure 1, the metal electrode becomes a barrier in the gas path that increases turbulence in the gas flow nearby. To ensure the homogeneity of the drift gas, a novel ion detector was proposed [14], and results showed that this structure can ensure the detection rate of ions and make the drift gas homogeneous.

3.3. Modelling in COMSOL Multiphysics. In simulations, it is assumed that the ion source and detector are ideal and that the drift gas flow is homogeneous. Thus, only the mass analyser and ions moving inside it are simulated. Ions are released from the start of the quadrupole analyser and travel under the combined action of the gas flow and quadrupole field. The ions are stable and can be detected if they reach the end of the analyser within a given period of time.

A quadrupole mass filter model based on rectangular electrodes was created. The length of the electrodes $L$ was $80 \mathrm{~mm}$, and the distance from the electrodes to the centre was $5 \mathrm{~mm}$. Because the analyser was within a closed structure, a drift tube was omitted.

In COMSOL Multiphysics, modelling for multiphysics problems is divided into three steps. First, the RF voltage $\phi_{0}=-(U+V \cos \omega t)$ was applied to the upper and lower electrodes, and $\phi_{0}=+(U+V \cos \omega t)$ was applied to the left and right electrodes. The electrostatics (es) interface and electric current $(\mathrm{ec})$ interface were used to calculate the electrostatic field and RF electric field, respectively. Second, the laminar flow (spf) interface was used to model the drift gas flow inside the analyser. The inlet boundary condition was set to be "laminar inflow," and the flow rate was $V_{0}$. The outlet boundary condition was set to be "pressure" with a value of $p$. Last, the computation results of the es, ec, and spf were coupled to the particle tracing for fluid flow (fpt) interface. At the entrance of the analyser, a circular region with a radius of $r_{\mathrm{src}}=0.5 \mathrm{~mm}$ was designated for ion release, and ions were released at $0 \mathrm{~ms}$. The mass of each ion was $m_{\mathrm{i}}$, and the charge was 1 . The initial velocity was along the $z$-direction, and the kinetic energy was $E_{\mathrm{i}}=5 \mathrm{eV}$. Ions were 

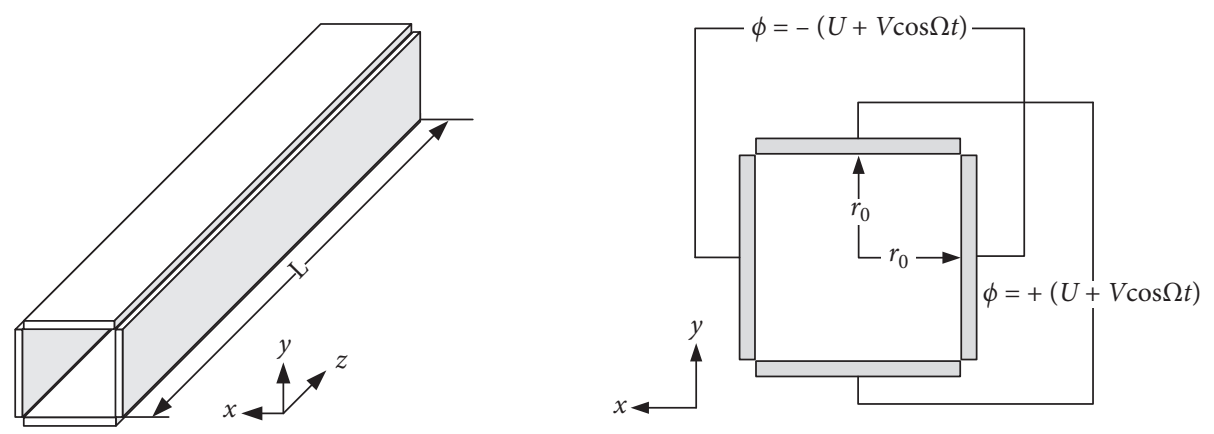

FIGURE 2: Schematic diagram of the quadrupole mass filter based on rectangular electrodes.

released 10 times in the first $\mathrm{RF}$ cycle, where 50 ions are released each time. A total of 500 ions were released in a single simulation. In addition, the purpose of this simulation was to verify the feasibility of the proposed method; thus, the voltage difference between the front and rear ends of the quadrupole was not set.

Because the ec, es, spf, and fpt interfaces were used, the calculation was divided into two steps. First, the electrostatic field and RF electric field are calculated in the stationary and frequency domains, respectively. Second, the time-dependent tracking of the ions was solved. Thus, ion trajectory was controlled by the electric field and gas flow.

The ion transmission probability is the ratio between the number of ions detected and the total number of ions, which describes the signal strength of the spectral peak. Under the same conditions, the larger the signal strength is, the higher the sensitivity of the instrument is.

Simulation parameters are shown in Table 1. A highfrequency RF voltage was used to prevent the stability regions' expansion in a low-vacuum environment $[7,15]$. Under this condition, the Mathieu parameter was $a=0$ and $q=0.95$, which were located within the stable regions. Therefore, all ions should pass through the mass filter and be detected.

\section{Simulation Results and Discussion}

4.1. Ion Motion without Drift Gas Flow. The step of the simulation was set to $0.1 / f$, and the length of the simulation time was set to $100 / f$. When the drift gas flow rate at the inlet was $V_{0}=0 \mathrm{~L} / \mathrm{min}$, there was no gas flow, and the environmental pressure $p$ changed. At the end of the simulation, the ion motion state under different pressures is shown in Figure 3, in which the colours describe the speed of ions. The simulation results show that when the pressure was $0 \mathrm{~Pa}$, nearly all ions can pass through the filter, and the transmission probability was 0.96 . As pressure increased, the transmission probability decreased gradually. When the pressure was $10 \mathrm{~Pa}$, the transmission probability was 0.05 and dropped to zero as the pressure increased further.

In this simulation, the working point of the Mathieu equation was located within the stable region. However, the transmission probability decreased as the pressure increased, which was caused by increased ion collisions with background gas molecules at higher pressure. In the $x-y$
TABLE 1: Simulation parameters for low-vacuum mass spectrometry.

\begin{tabular}{lc}
\hline Parameters & Value \\
\hline Length of the mass filter $(L)$ & $80 \mathrm{~mm}$ \\
Radius of the mass filter $\left(r_{0}\right)$ & $5 \mathrm{~mm}$ \\
Ion mass $\left(m_{\mathrm{i}}\right)$ & $40 \mathrm{amu}$ \\
Background gas molecular mass $\left(m_{\mathrm{g}}\right)$ & $10 \mathrm{amu}$ \\
Background pressure $(p)$ & -- \\
Drift flow rate at inlet $\left(V_{0}\right)$ & $0 \mathrm{~L} / \mathrm{min}$ \\
Initial kinetic energy of ions $\left(E_{\mathrm{i}}\right)$ & $5 \mathrm{eV}$ \\
DC component of RF voltage $(U)$ & $85 \mathrm{~V}$ \\
Amplitude of RF voltage $(V)$ & $1080 \mathrm{~V}$ \\
Frequency of RF voltage $(\omega)$ & $4 \mathrm{MHz}$ \\
\hline
\end{tabular}

direction, the ion trajectories gradually decreased and finally focused on the center of the mass analyser. In the $z$-direction, the ion kinetic energy was insufficient due to collision and finally remained in the interior of the mass analyser.

4.2. Ion Motion under Drift Gas Flow. To solve the problem of insufficient kinetic energy for ion motion in the $z$-direction under a low vacuum, a method was proposed whereby the drift gas flow imparts energy for ion motion. The outlet pressure of the mass analyser was set as $p=150 \mathrm{~Pa}$, and the remaining parameters were the same as in Table 1. The flow rate of the drift gas was also changed. The corresponding transmission probability is shown in Figure 4, and the ion states at the end of the simulation are shown in Figure 5.

In Figure 4, as the gas flow rate increased, the transmission probability gradually increased. When the gas flow rate was greater than $6 \mathrm{~L} / \mathrm{min}$, the probability was stable and fluctuated around 0.8. In Figure 5, the ions were still located in the inlet area of the mass analyser at the end time of simulation when the flow rate was $0 \mathrm{~L} / \mathrm{min}$, and the transmission probability was 0 . At a flow rate of $2 \mathrm{~L} / \mathrm{min}$, the transmission probability was still 0 ; however, the ions moved farther. When the flow rate was increased to $6 \mathrm{~L} / \mathrm{min}$, nearly all the ions passed through the mass filter and reached the detector. Few ions stopped at the inner wall of the electrodes due to the higher-order electric field and collisions with background gas molecules. The simulation results showed that the drift gas flow imparted sufficient kinetic energy to 


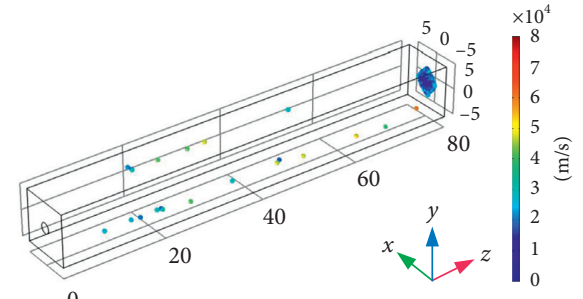

(a)

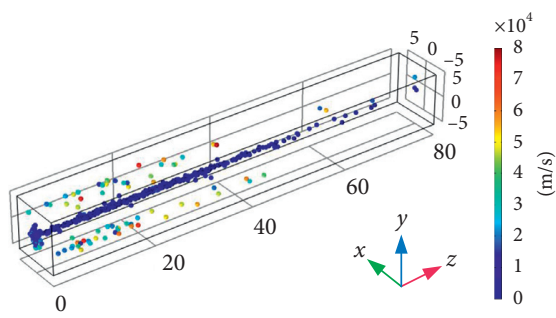

(c)

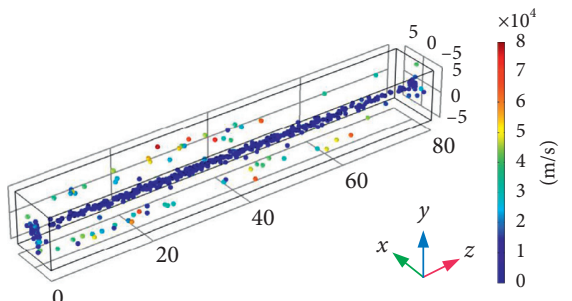

(b)

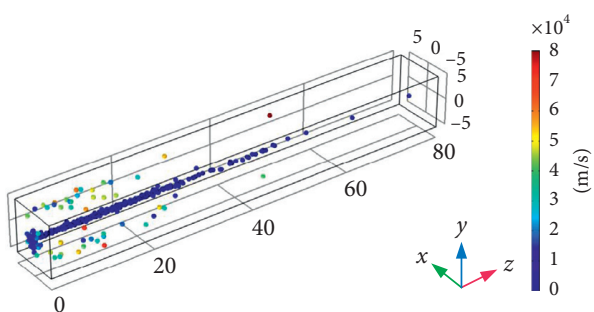

(d)

FiguRE 3: Ion states under different background pressures without drift gas flow: (a) $p=0 \mathrm{~Pa},(\mathrm{~b}) p=10 \mathrm{~Pa},(\mathrm{c}) p=20 \mathrm{~Pa}$, and (d) $p=30 \mathrm{~Pa}$.

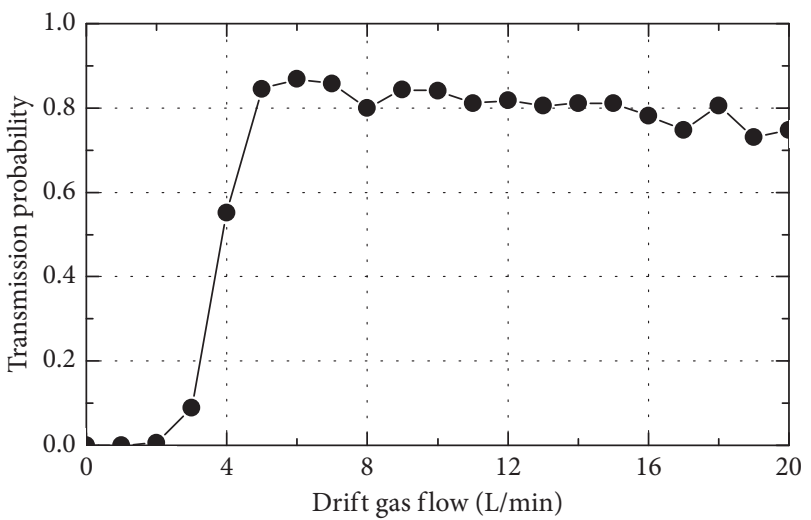

FIGURE 4: Transmission probability under different gas flow rates when the pressure was $150 \mathrm{~Pa}$.

the ions for $z$-direction motion, allowing the ions to reach the detector even under relatively high pressure.

Figure 6 shows a comparison of the transmission probability in the absence of drift gas flow and under a gas flow rate of $10 \mathrm{~L} / \mathrm{min}$ when the pressure varied from 0 to $300 \mathrm{~Pa}$. As pressure increased, the transmission probability decreased to 0 eventually under both conditions. However, in the absence of the drift gas flow, when the pressure reached $15 \mathrm{~Pa}$, the probability decreased to 0 . Conversely, the pressure was $250 \mathrm{~Pa}$ when the gas flow rate was $10 \mathrm{~L} / \mathrm{min}$. Considering the transmission probability to be 0.2 as the criterion for mass analysis, the maximum pressure can be increased from $6 \mathrm{~Pa}$ to $225 \mathrm{~Pa}$ for mass analysis by introducing the gas flow.

In addition, when the pressure was below approximately $175 \mathrm{~Pa}$, the transmission probability first decreased and then increased as pressure increased because the pressure and flow rate cannot be discussed separately even though the pressure and gas flow rate can be set separated in multiple physical simulations. Pressure describes the number of gas molecules in a space: when pressure was too low, the number of background gas molecules decreased, and a higher flow rate could not be achieved, limiting the kinetic energy transferred from the background gas flow to the sample ions. When the pressure increased, the pushing effect of the gas flow on the sample ions increased, and the probability increased.

4.3. Spectra Obtained under High Pressure. With the slope of the mass-scan line equal to $k=a / q=2 U / V=1 / 3$ and the other parameters equal to those in Table 1, Figure 7 shows the spectra obtained under three different simulation conditions for the ion with $m_{\mathrm{i}}=40 \mathrm{amu}$. The spectrum numbered (1) was obtained under vacuum conditions (i.e., no gas flow was present). A clear spectral peak was obtained at $m /$ $z=40$, and the maximum transmission probability was approximately 0.46 . The spectrum numbered (2) was obtained under a pressure of $p=150 \mathrm{~Pa}$ with no gas flow. Results show that the ions did not have sufficient kinetic energy to move in the $z$-direction and thus could not reach the detector due to the effect of background gas molecules. Consequently, the transmission probability was zero, and no peak was generated. The spectrum numbered (3) was obtained under a pressure of $p=150 \mathrm{~Pa}$ with a gas flow. In spectrum (3), a spectrum peak was generated at $m / z=40$; however, the peak was weaker and wider than that generated under vacuum.

These simulation results show that the proposed method using the drift gas can impart kinetic energy to ion motion in the $z$-direction. The problem of ions not reaching the detector due to a lack of kinetic energy under high pressure was thus solved. Although the spectra obtained were weaker than the results under vacuum in terms of peak intensity and width, the spectra were markedly better than the result 


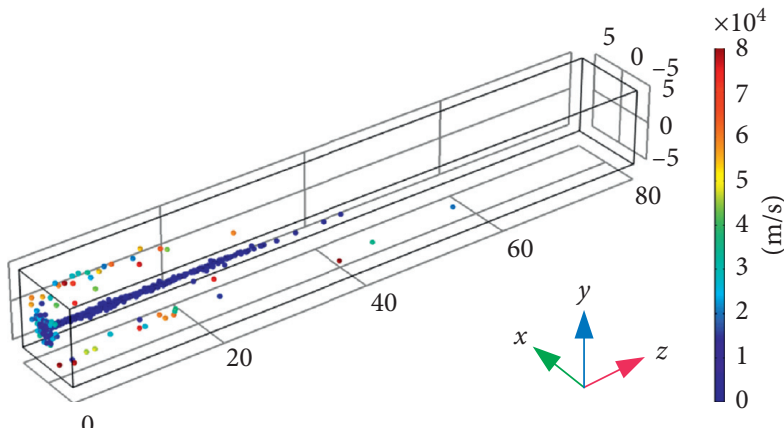

(a)

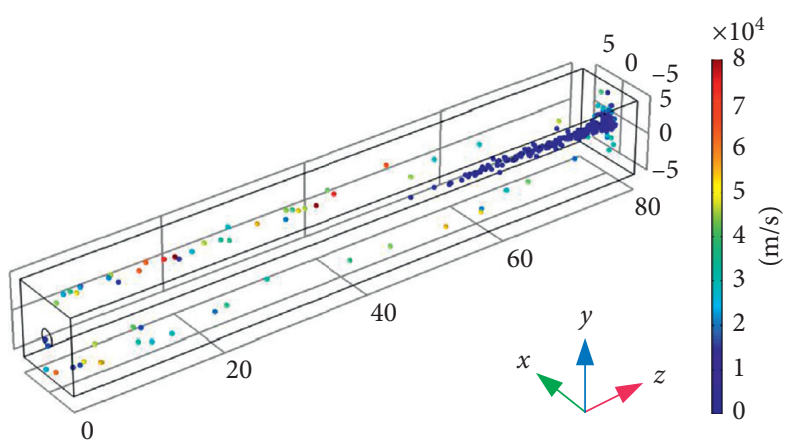

(c)

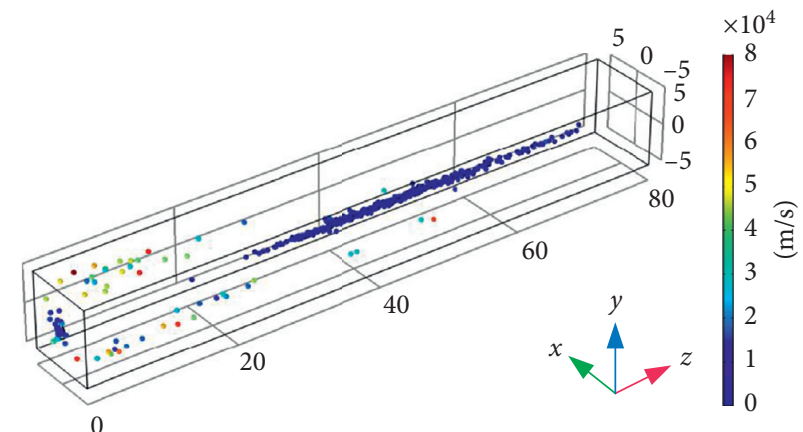

(b)

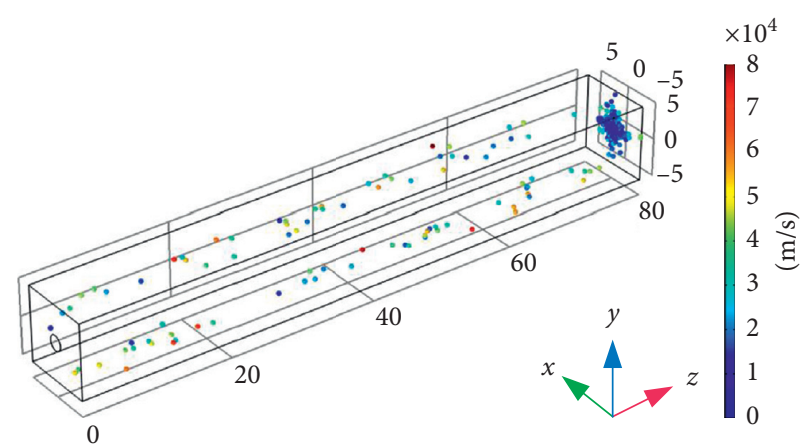

(d)

Figure 5: Ion states at the end of the simulation under different gas flow rates when the pressure was $150 \mathrm{~Pa}:(\mathrm{a}) V_{0}=0 \mathrm{~L} / \mathrm{min},(\mathrm{b}) V_{0}=2 \mathrm{~L} /$ $\min$, (c) $V_{0}=4 \mathrm{~L} / \mathrm{min}$, and (d) $V_{0}=6 \mathrm{~L} / \mathrm{min}$.

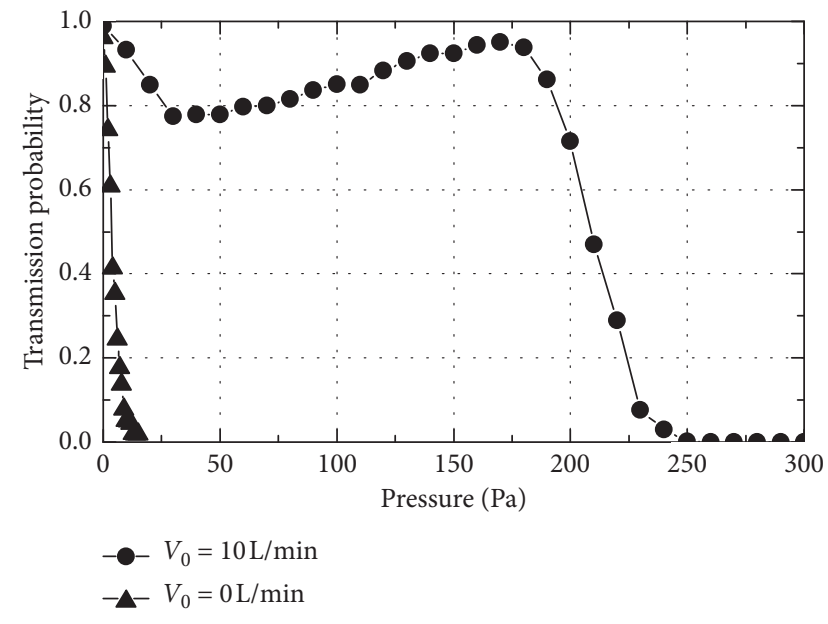

FIGURE 6: Comparison of the transmission probability when $V_{0}$ was $10 \mathrm{~L} / \mathrm{min}$ and $0 \mathrm{~L} / \mathrm{min}$.

without gas flow. Consequently, the proposed method is suitable when high precision is not required.

\section{Experimental Validation}

5.1. Vacuum System and Ion Source. The vacuum system was modified to achieve the drift gas flow, and its block diagram is shown in Figure 8. First, a flow guide was installed between the rear of the mass analyser and the vacuum pump. Second, an inlet hole for the background gas was placed in the front of the mass analyser, and a needle valve was mounted on the gas path.

The working principle of the electron ionisation (EI) source is similar to an incandescent light bulb. Relevant experiments have shown that the EI source could work in a low-vacuum environment with an inert background gas. Therefore, the EI source was used as the ion source for this experiment.

5.2. Mass Analyser and Drift Tube. To build the mass analyser, four printed circuit boards (PCBs) were used as rectangular electrodes, on which the top and bottom layers were electrically connected by vias, as shown in Figure 9(a).

As described above, the drift tube is a tubular structure with two ends open. The machined drift tube is shown in Figure 9(b) and was fabricated with a $3 \mathrm{D}$ printer using polylactic acid (PLA). Four holes were left on each side to connect electrical wiring, and slide rails were designed inside the drift tube to install the plate electrodes.

5.3. Ion Detector. A double-layer plate structure in the ion detector should be used to ensure homogeneous gas flow. As shown in Figure 10, the detector was composed of two PCBs that acted as electrodes and the corresponding fixed structures fabricated by the 3D printer. Each electrode 


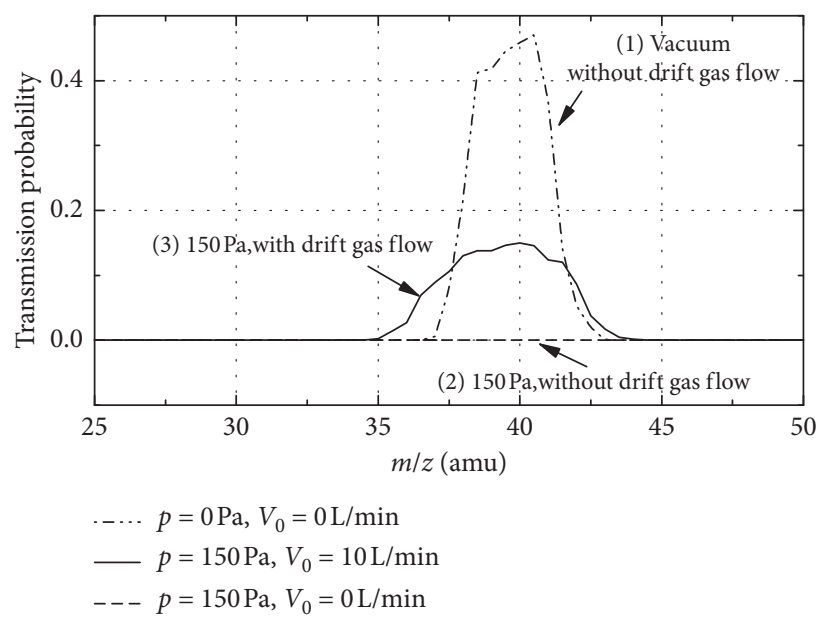

Figure 7: Mass spectra under different pressures and gas flow rates.

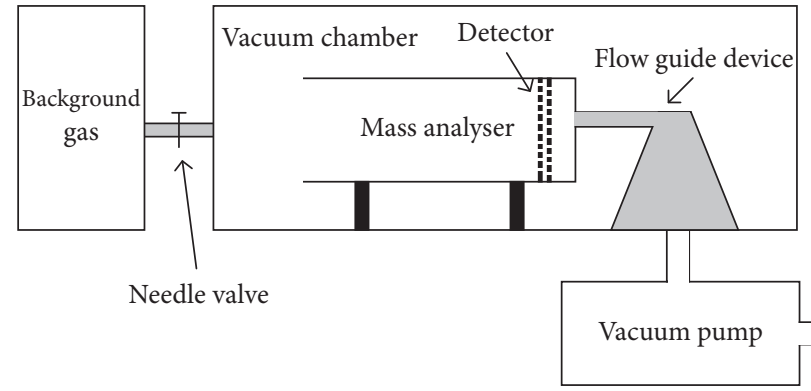

FIGURE 8: Block diagram of a modified vacuum system.

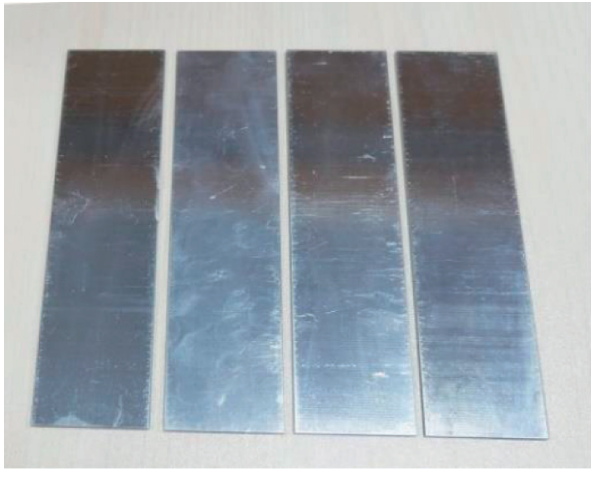

(a)

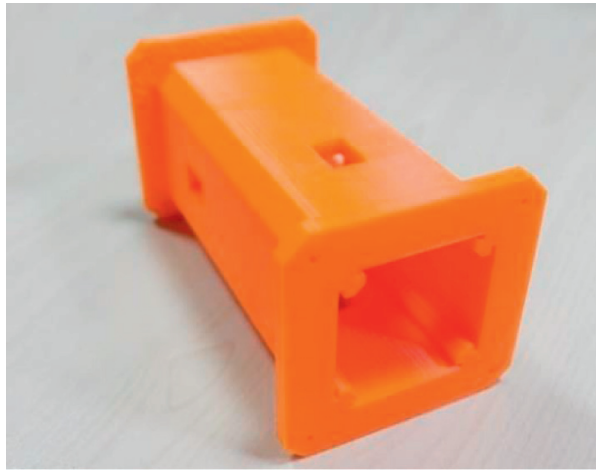

(b)

FIGURE 9: (a) Electrodes customised by the printed circuit board and (b) gas flow tube fabricated by the 3D printer.

contained many staggered small holes through which the gas could pass into the detector. The spacing between the holes was $100 \mathrm{mil}$, and the diameter of the holes was $50 \mathrm{mil}$. However, the two electrodes were electrically connected by an electrical wire to ensure they were equipotential.

5.4. Component Assembly and Electrical System. The EI source, mass analyser, drift tube, and ion detector were assembled to comprise the core components of the mass analysis experiment, as shown in Figure 11.

The electrical system used for mass analysis consisted of a current source, a signal generator (Agilent, 33250A), a power amplifier (E\&I, 1040L), a voltage amplifier, a picoammeter (Keithley 478), and several DC power sources. The circuit connection diagram is shown in Figure 12. In the EI source, the current source exhibited a steady current to the filament, and a DC power source exhibited a bias voltage 


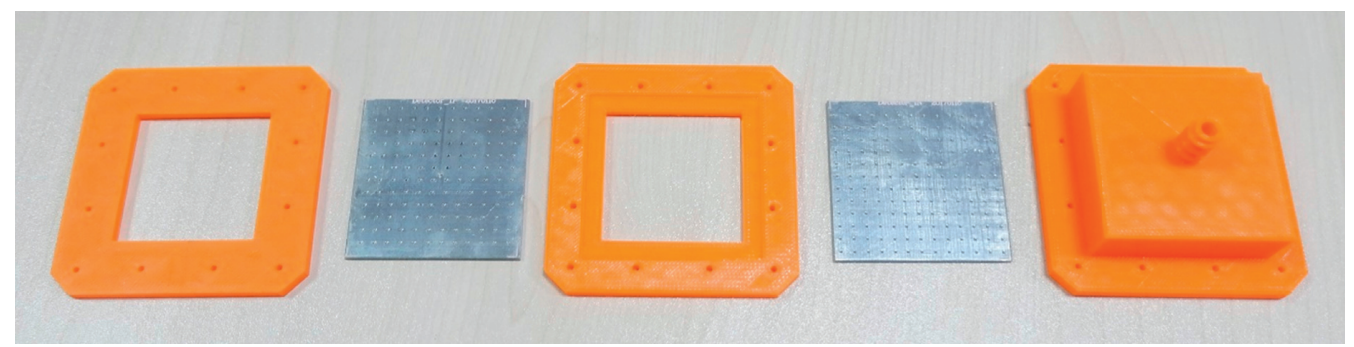

FIGURE 10: Ion detector customised with printed circuit boards and fixed structure fabricated by 3D printing.

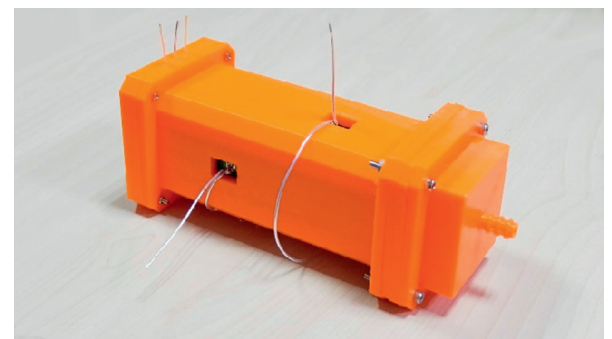

FIGURE 11: Mass analysis device used for high-pressure mass spectrometry.

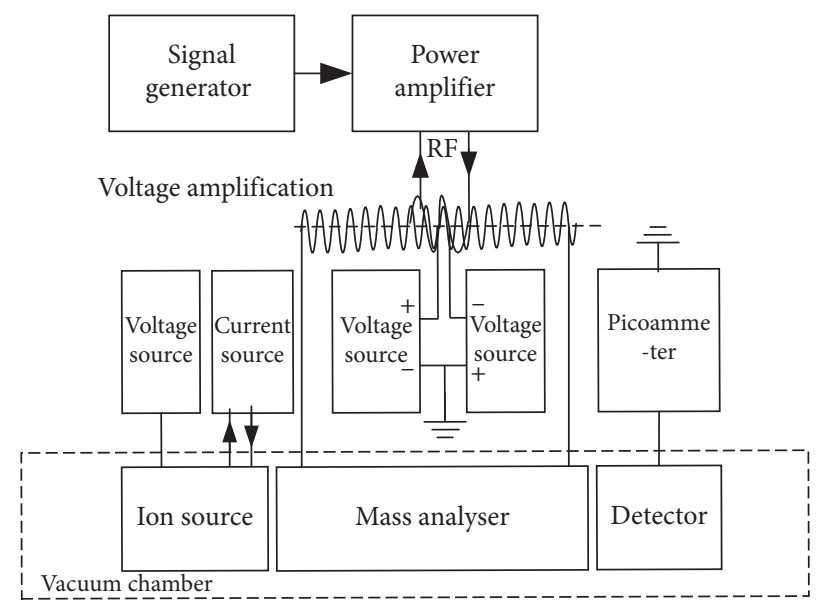

FIGURE 12: Connections in the electrical system used for low-vacuum mass spectrometry.

on the repeller. For RF voltage generation, the signal generator produced a low-voltage RF signal, which was then amplified through the power amplifier and a voltage amplifier to create the high-voltage RF signal for the mass analyser. In the ion detector, a picoammeter was connected to the ion detector to measure the ion current. The current described the state of ion movement in the mass analyser.

5.5. Experimental Results and Analysis. The mass analysis device was installed in the vacuum chamber, and helium was used as the background gas. The vacuum pump was turned on, and the pressure in the chamber was accurately controlled by the needle valve to maintain the pressure in the chamber at $20 \mathrm{~Pa}$.
Due to the limitations of experimental conditions and the machining precision of the mass analyser, we selected several operational points to identity the state of ion movement via the picoammeter instead of using mass scanning. For the spectral peak $m / z=92$ of methyl salicylate, the selected operation points $(a, q)$ in the stable region were identified by adjusting the $U$ - and $V$-values and are shown in Figure 13. Measurement results are shown in Table 2.

The experimental results show that when the pressure was $20 \mathrm{~Pa}$, the measured current was on the order of $10^{-3} \mathrm{nA}$ if the traditional method was used, regardless of if the operational point was located inside or outside the stable region. This value was nearly identical to that of the static noise, indicating that effectively zero ions reached the detector (i.e., the mass analysis failed). However, with the 


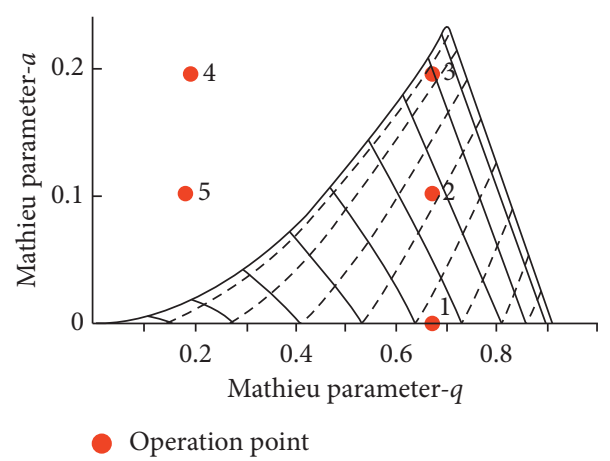

FIGURE 13: Location of operation points relative to a stable region.

TABLE 2: Experimental results obtained at $20 \mathrm{~Pa}$.

\begin{tabular}{|c|c|c|c|c|c|}
\hline \multirow{2}{*}{ No. } & \multirow{2}{*}{$a$ value } & \multirow{2}{*}{$q$ value } & \multirow{2}{*}{ Operation point } & \multicolumn{2}{|c|}{ Picoammeter current $(\mathrm{nA})$} \\
\hline & & & & Traditional method (no gas flow) & Proposed method (with gas flow) \\
\hline 1 & 0 & 0.66 & Inside the stability region & 0.003 & 20.1 \\
\hline 2 & 0.1 & 0.66 & Inside the stability region & 0.005 & 16.5 \\
\hline 3 & 0.2 & 0.66 & Inside the stability region & 0.005 & 12.0 \\
\hline 4 & 0.2 & 0.2 & Outside the stability region & 0.004 & 1.8 \\
\hline 5 & 0.1 & 0.2 & Outside the stability region & 0.004 & 3.6 \\
\hline
\end{tabular}

proposed method, the measured currents at operational points 1,2 , and 3, which were inside the stable region, were significantly higher than those at operational points 4 and 5 , which were outside the stable region. These results show that the ions can be separated by introducing a drift gas flow at a pressure of $20 \mathrm{~Pa}$.

\section{Conclusion}

Performing mass spectrometry (MS) in a low-vacuum environment can substantially reduce the cost, size, weight, and power of the instrument. With quadrupole mass filter at higher pressures, the lack of kinetic energy along the ions' axial direction of motion prohibits mass analysis. Thus, a mass analysis method was proposed in this study where a drift gas flow was used to impart ejection energy to the ions. Simulations in COMSOL Multiphysics showed that the proposed method is valid.

A simplified experimental platform was then built to apply mass spectrometry at a spectral peak $\mathrm{m} / z$ of 92 for methyl salicylate. The results measured by using a picoammeter showed that the currents at operational points inside the stable region were significantly higher than those outside the stable region, which showed that the ions can be separated in a high-pressure environment using the proposed method.

In this experiment, the analysis results at several operational points were also meaningful because the line is made of points, despite mass scan not being performed. However, what degree of resolution can be achieved and how to improve it must be studied in future work.

\section{Data Availability}

The data used to support the findings of this study are available from the corresponding author upon request.

\section{Conflicts of Interest}

The authors declare that they have no conflicts of interest.

\section{Acknowledgments}

This work was supported by the National Natural Science Foundation of China (21505028) and the Natural Science Foundation of Shandong Province, China (ZR2014FM023 and ZR2015BQ004).

\section{References}

[1] K. H. Blakeman, C. A. Cavanaugh, W. M. Gilliland, and J. M. Ramsey, "High pressure mass spectrometry of volatile organic compounds with ambient air buffer gas," Rapid Communications in Mass Spectrometry, vol. 31, no. 1, pp. 27-32, 2017.

[2] K. H. Blakeman, D. W. Wolfe, C. A. Cavanaugh, and J. M. Ramsey, "High pressure mass spectrometry: the generation of mass spectra at operating pressures exceeding 1 Torr in a microscale cylindrical ion trap," Analytical Chemistry, vol. 88, no. 10, pp. 5378-5384, 2016.

[3] L. Gao, Q. Song, G. E. Patterson, R. G. Cooks, and Z. Ouyang, "Handheld rectilinear ion trap mass spectrometer," Analytical Chemistry, vol. 78, no. 17, pp. 5994-6002, 2006.

[4] L. Gao, A. Sugiarto, J. D. Harper, R. G. Cooks, and Z. Ouyang, "Design and characterization of a multisource hand-held 
tandem mass spectrometer," Analytical Chemistry, vol. 80, no. 19, pp. 7198-7205, 2008.

[5] L. Li, T.-C. Chen, Y. Ren, P. I. Hendricks, R. G. Cooks, and Z. Ouyang, "Mini 12, miniature mass spectrometer for clinical and other applications-introduction and characterization," Analytical Chemistry, vol. 86, no. 6, pp. 2909-2916, 2014.

[6] Y. Zhai, Y. Feng, Y. Wei, Y. Wang, and W. Xu, "Development of a miniature mass spectrometer with continuous atmospheric pressure interface," The Analyst, vol. 140, no. 10, pp. 3406-3414, 2015.

[7] P. Jiang, Z. Zhou, Z. Wu, and Z. Zhao, "Low-vacuum cylindrical ion trap mass spectrometry," Instrumentation Science and Technology, vol. 46, no. 6, pp. 614-627, 2018.

[8] M. He, D. Guo, Y. Feng et al., "Realistic modelling of ionneutral collisions in quadrupole ion traps," Journal of Mass Spectrometry, vol. 50, no. 1, pp. 95-102, 2015.

[9] I. V. Chernushevich and B. A. Thomson, "Collisional cooling of large ions in electrospray mass spectrometry," Analytical Chemistry, vol. 76, no. 6, pp. 1754-1760, 2004.

[10] W. Xu, Q. Song, S. A. Smith, W. J. Chappell, and Z. Ouyang, "Ion trap mass analysis at high pressure: a theoretical view," Journal of the American Society for Mass Spectrometry, vol. 20, no. 11, pp. 2144-2153, 2009.

[11] Q. Song, W. Xu, S. A. Smith et al., "Ion trap mass analysis at high pressure: an experimental characterization," Journal of Mass Spectrometry, vol. 45, no. 1, pp. 26-34, 2010.

[12] R. E. March, "An introduction to quadrupole ion trap mass spectrometry," Journal of Mass Spectrometry, vol. 32, no. 4, pp. 351-369, 1997.

[13] P. Jiang, Z. Zhou, S. Wu, and Z. Zhao, "A novel quadrupole mass filter based on rectangular electrodes," in Proceedings of the International Conference on Instrumentation and Measurement, Computer, Communication and Control (IMCCC 2018), pp. 1524-1527, Harbin, China, July 2018.

[14] P. Jiang, Z. Zhou, and Z. Zhao, "A novel ion detector aiming at the homogeneous of drift gas," International Journal for Ion Mobility Spectrometry, vol. 20, no. 3-4, pp. 111-117, 2017.

[15] P. Jiang, Z. Zhou, H. Chen, and Z. Zhou, "Numerical method for stability region in quadrupole at low vacuum [J]," Journal of Harbin Institute of Technology, vol. 49, no. 5, pp. 31-35, 2017, In Chinese. 